\section{THU0303 THE EARLY PSORIATIC ARTHRITIS SCREENING QUESTIONNAIREIDENTIFIES PATIENTS WITH PSORIATIC ARTHRITIS AMONGST TREATED PATIENTS WITH PSORIASIS}

\begin{abstract}
K. Nadeau' ${ }^{1}$ F. Briggs ${ }^{2,3}$, S. O'Neill ${ }^{2,3}$, D. Sumpton ${ }^{2,3}$, G. Cains ${ }^{4}$, J. Woods ${ }^{4}$, ${ }^{2,3}$ D. Chessman. ${ }^{1}$ Université Laval, CHU de Québec, Québec, Canada; ${ }^{2}$ Department of Rheumatology, Liverpool Hospital; ${ }^{3}$ Ingham Research Institute, The University of New South UK; ${ }^{4}$ Department of Dermatology, Liverpool Hospital, Sydney, Australia
\end{abstract}

Background: Studies suggest a high prevalence (approximately 15\%) of undetected psoriatic arthritis (PsA) amongst patients with psoriasis ${ }^{1}$. A number of screening questionnaires have been designed to allow detection of such patients. This includes the Early Psoriatic Arthritis Screening Questionnaire (EARP) which detects early PSA in untreated patients with psoriasis, with a sensitivity of $85.2 \%$ and specificity of $91.6 \%{ }^{2}$. Little is known about whether such questionnaires are also able to detect PsA in treated patients with psoriasis.

Objectives: To determine the case finding ability of EARP in a tertiary centre cohort of treated psoriasis patients.

Methods: All patients attending a tertiary centre psoriasis clinic were invited to complete the EARP. EARP comprises a 10 point patient reported questionnaire regarding symptoms of joint disease. Scores of 3 or more are considered positive. All patients who completed the questionnaire and received a positive score were assessed by a rheumatologist. Diagnosis of PsA was made by clinician impression and CASPAR criteria. Disease activity was assessed using psoriasis area severity index (PASI), 66/68 swollen and tender joint count, SPARCC enthesitis index, CRP and Health associated quality of life disability index (HAQ-DI). The composite disease activity measure DAPSA and the OMERACT definition of minimal disease activity were determined.

Results: 133 patients were invited to complete the EARP questionnaire and 119 participated. Fifty patients had a positive result (42\%). Of these, 8 were known to have PsA and under rheumatologic care. A further 21 attended for formal rheumatologic assessment. Thirteen of the 21 patients $(61.9 \%)$ were found to have psoriatic arthritis and were not under the care of a rheumatologist. This represents $10 \%$ of the initial 133 patients screened. Ten of those patients were further assessed. The average age was 52.8 and BMI 33.2. Seven patients were male. All 10 were on biologic agents but only 3 on concurrent conventional DMARDs. Average tender joint count was 16, swollen joint count 3.6, SPARCC 6.2 and PASI score 3.42. Only 1 patient was in minimal disease activity.

Conclusions: The EARP tool can identify patients with active PsA amongst patients with psoriasis, even those on treatment with biologic agents. Such a tool may be useful in identifying patients who may benefit from rheumatology care.

\section{REFERENCES:}

[1] Villani AP, Rouzaud M, et al. Prevalence of undiagnosed psoriatic arthritis among psoriasis patients: Systematic review and meta-analysis.J Am Acad Dermatol 2015;73(2):242-8

[2] Tinazzi I, Adami S, Zanolin EM, et al. The early psoriatic arthritis screening questionnaire: a simple and fast method for the identification of arthritis in patients with psoriasis. Rheumatology 2012;51(11):2058-63.

Disclosure of Interest: None declared

DOI: 10.1136/annrheumdis-2018-eular.5168

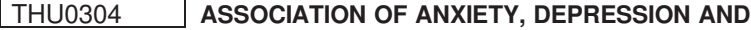 FATIGUE WITH DISEASE ACTIVITY, JOINTS EROSION AND SKIN LESION SEVERITY IN EARLY PSORIATIC ARTHRITIS PATIENTS}

E.E. Gubar, E.Y. Loginova, A.D. Koltakova, S.I. Glukhova, T.V. Korotaeva. V.A. Nasonova Research Institute of Rheumatology, Moscow, Russian Federation

Background: Depression is one of the precursors of psoriasis and psoriatic arthritis (PSA) development. It was found that depression and anxiety negatively affect the achievement of remission in PsA. B. Michelsen 2017] Interrelation of anxiety, depression and fatigue (according to patient-reported outcomes) with disease activity, erosive arthritis and skin lesion severity in early PsA hadn't been sufficiently studied.

Objectives: To study anxiety, depression and fatigue disorders (according to patient-reported outcomes) and their correlation with disease activity, erosive arthritis and severity of psoriasis in early PsA patients (pts).

Methods: 78 pts (M/F-39/39) with early PsA according to CASPAR criteria were included; all pts had peripheral arthritis for $\leq 2$ years; mean age $36.5 \pm 10.7$ years, disease duration $12.2 \pm 10.3 \mathrm{mo}$. It was a treatment naïve cohort. All pts underwent standard clinical examination of PsA activity. Mean disease activity indexes $(D A S)=4.0 \pm 1.4, D A S 28=4.2 \pm 1.1 .78$ patients were studied for fatigue (according to FACIT), patient global disease activity (PGA), patients pain measured by VAS and Health Assessment Questionnaire (HAQ); 66 patients (M/F-33/33) were studied for anxiety and depression (according to HADS). At HADS score $\geq 8$ patients had anxiety and depression disorders. Higher scores for FACIT scales indicate better quality of life (less fatigue). Skin lesion severity was evaluated in terms of body surface area (BSA) affected and Psoriasis Area Severity Index (PASI). When BSA was $\geq 3 \%$, PASI was calculated. PASI $\geq 11$ indicates moderate and severe psoriasis. Descriptive statistics was used, $M \pm S D, M e$ [Q25;Q75], Utest were performed; $p<0.05$ was considered to indicate statistical significance.

Results: Mean FACIT score was low amounting to $35.3 \pm 9.6$, testifying increased fatigue; mean anxiety index was $5.7 \pm 3.1$, depression index was $3.8 \pm 3.0$. Anxiety disorders were detected in 16 out of $66(24.2 \%)$ pts, depression disorders in 9 out of $66(13.0 \%)$ pts. Negative correlation was found between FACIT score and DAS $(r=-0.26)$, DAS28 $(r=-0.26)$, CRP $(r=-0.27)$, PGA $(r=-0.35)$; and pain VAS $(r=-$ $0.25)$. Depression was more pronounced in pts with erosive arthritis in hands and or feet $(r=0.31)$. Negative correlation of FACIT score $(r=-0.54)$, correlation of anxiety $(r=0.26)$ and depression $(r=0.33)$ indexes was found with health-related functional indexes according to HAQ. HADS indexes (anxiety and depression) are cross-correlating $(r=0.51)$ and are negatively correlating with FACIT scores $(r=$ 0.49 and $r=-0.48$, accordingly). An association was found of anxiety and depression indexes with the severity of psoriasis PASI index $(r=0.38$ and $r=0.31$, accordingly).

Conclusions: In early treatment-naïve PsA patients, increased fatigue and in a quarter of cases anxiety disorders, in $13 \%$ of patients depression disorders had been found. Psychological disorders are associated with PsA activity, the severity of psoriasis and joints erosion. Fatigue, anxiety and depression in early PsA patients result in the reduction of their functional capacity.

Disclosure of Interest: None declared

DOI: 10.1136/annrheumdis-2018-eular.2404

\section{THU0305 MINIMAL DISEASE ACTIVITY (MDA) ATTAINMENT AFTER STARTING BIOLOGICAL (B) DMARDS AND NON- BDMARDS TREATMENT IN PSORIATIC ARTHRITIS PATIENTS (PTS) IN ROUTINE CARE: RUSSIAN PSORIATIC ARTHRITIS REGISTRY (RU-PSART) DATA}

${ }^{1}$ E. Loginova, T. Korotaeva ${ }^{1}$, A. Koltakova ${ }^{1}$, E. Gubar ${ }^{1}$, Y. Korsakova ${ }^{1}$, E. Nasonov ${ }^{1}$, A. Lila ${ }^{1}$, M. Sedunova ${ }^{2}$, T. Salnikova ${ }^{3}$, I. Umnova ${ }^{4}$, I. Bondareva ${ }^{5}$ U. Zagidulina ${ }^{6}$, P. Zemtsova ${ }^{7}$ on behalf of the RU-PsART study group. ${ }^{1}$ Nasonova Research Institute of Rheumatology, Moscow, ${ }^{2}$ St. Petersburg Clinical Rheumatology Hospital No.25, St. Petersburg; ${ }^{3}$ Tula Regional Hospital, Tula ${ }^{4}$ Omsk Regional Hospital, Omsk; ${ }^{5}$ Kemerovo Regional Hospital, Kemerovo; ${ }^{6}$ Kazan City Hospital No.7, Kazan; ${ }^{7}$ Nizhny Novgorod Regional Clinical Hospital $n$. a. Semashko, Nizhny Novgorod, Russian Federation

Background: MDA is a valid instrument for evaluating PsA treatment results. There is limited data about MDA attainment after starting bDMARDs and nonbDMARDs in routine care. RU-PSART collected data from 25 rheumatology clinics in the Russian Federation.

Objectives: evaluate MDA attainment after starting bDMARDs and nonbDMARDs treatment in PsA pts in routine care.

Methods: 294 (M/F-133/161) pts with PsA, diagnosed according to CASPAR criteria, mean age 41.2 \pm 1.9 (Min $21-$ Max 72) years (yrs.), PsA duration 6.1 \pm 5.3 (Min 0 - Max 31) yrs., psoriasis duration 13.6 \pm 10.7 (Min 0.2 - Max 54.8) yrs. were included in the RU-PSART after signing consent participation forms. The present analysis included 274 pts who have data concerning PsA activity, treatment and MDA. The number of pts who reached MDA at least once were calculated. At the time of evaluation 81 out of 274 pts (29.6\%) were taking bDMARDs \pm sDMARDs Infliximab (25 pts), Etanercept (16 pts), Adalimumab (14pts), Ustekinumab (8pts) Golimumab (5pts), Sekukinumab (2pts). 193 out of 274 pts $(70.4 \%)$ were taking other types of treatment - sDMARDs \pm NSAID, mostly methotrexate $(74.2 \%)$, sulfasalazine $(12 \%)$, leflunomide $(3.6 \%)$, hydroxichlorochine $(0.4 \%)$; steroids $(9.8 \%)$. All pts underwent evaluation of PsA activity by DAS28, CRP, Pt/Physician GA, Pain GA by VAS (0-100 mm), swollen/tender joints count (SJC/TJC), DAPSA and considered REM $\leq 4 / L D A \leq 14 . M \pm S D, M e$ [Q25; Q75], Min-Max,\%, U-test, ORs with $95 \% \mathrm{Cl}$ were performed. All $\mathrm{Cl}>1, \mathrm{p} \leq 0.05$ were considered to indicate statistical significance.

Results: At time of evaluation 60 out of 274 pts $(21 \%)$ reached MDA at least once. Mean duration of sDMARDs and bDMARDs \pm sDMARDs was $11^{6: 17} /$ Min 3 Max 204 months and $9^{7: 15} /$ Min 2 - Max 82 months accordingly. 28 out of 193 pts $(10.4 \%)$ taking sDMARDs achieved MDA. Among 81 pts taking bDMARDs \pm sDMARDs MDA was seen in significantly more cases - 32 pts $(30.8 \%)$, $\mathrm{OR}=3.85[\mathrm{Cl} 95 \% 2.11-7.01]$. REM/LDA by DAPSA was found in significantly more cases compared to pts taking other therapies - in 50 out of 81 pts $(61.7 \%)$ and in 56 out of 193 pts $(29 \%)$ accordingly ( $\leq \leq 0.05$, U-test). Pts who had eve 